ISSN 1991-8631

Original Paper

http://indexmedicus.afro.who.int

\title{
Régime alimentaire de Scomberomorus tritor (Cuvier, 1831) dans le Golfe de Guinée
}

\author{
C. N'guessan DIAHA*, Konan N'DA et Yaya SORO \\ Laboratoire de Biologie et de Cytologie Animales (Unité de Formation et de Recherches en \\ Sciences de la Nature) Université d'Abobo Adjamé, 02 BP 801, Abidjan, Côte d'Ivoire. \\ *Auteur correspondant, E-mail: diahaconstance@yahoo.fr
}

\section{RESUME}

Scomberomorus tritor est une espèce de la famille des Scombridae débarquée quotidiennement au port d'Abidjan par les chaluts doubles. Le régime alimentaire de cette espèce de thonidés mineurs capturée par les chaluts pélagiques est étudié en fonction de la taille des spécimens et des saisons marines. Les estomacs de 832 individus (14 - $114 \mathrm{~cm}$ de longueur à la fourche), capturés de janvier 2006 à janvier 2008 ont été analysés. Sur le total des estomacs examinés, 612 contenaient des proies et 220 étaient vides, ce qui correspond à un coefficient de vacuité annuel de $26,44 \%$. Ce coefficient varie en fonction des saisons marines et de la taille des spécimens. L'indice d'importance relative (\% IRI) combinant les différents pourcentages (d'occurrence, numérique et pondérale) et le coefficient alimentaire (Q) ont été utilisés pour analyser l'importance des différents items identifiés. Au total, dix catégories d'aliments repartis en quatre groupes ont été identifiées. Scomberomorus tritor se nourrit principalement de Clupeidae (Sardinella maderensis (Lowe, 1839)) et d'Engraulididae (Engraulis encrasicolus (Linnaeus, 1758)) et accessoirement de Carangidae (Caranx senegalus (Cuvier, 1833)), d'Octopodidae (Octopus defilippi (Verny, 1851)) et de Sepiidae (Sepia elegans (Blainville, 1827)). C'est une espèce qui adapte son régime alimentaire en fonction des saisons marines en relation avec la disponibilité des individus proies. Par ailleurs, la proportion des items change avec la taille du poisson en rapport avec la stratégie alimentaire.

(C) 2010 International Formulae Group. All rights reserved.

Mots clés: Clupeidae, Scomberomorus tritor, alimentation, Golfe de Guinée.

\section{INTRODUCTION}

Scomberomorus tritor (Cuvier, 1831) (S. tritor) se rencontre dans l'Atlantique Ouest le long de la côte des États-Unis, depuis le Cap Cod jusqu'à Miami. Dans le golfe du Mexique, cette espèce se distribue depuis la Floride jusqu'à Yucatan (Johnson et Gill, 1998). C'est un poisson épipélagique qui apparaît de façon saisonnière dans les eaux côtières. Dans l'Atlantique Est, cette espèce est concentrée dans les îles Canaries, de Dakar à l'Angola et particulièrement dans le golfe de Guinée (Collette et Nauen, 1983).

S. tritor appartient à la famille des Scombridés et particulièrement au groupe des thonidés mineurs (Collette et al., 2001; Johnson et Gill, 1998). Elle joue un rôle important dans l'économie de la Côte d'ivoire, compte tenu de l'importance des débarquements annuels et des emplois 
annexes que sa pêche génère. Une quantité de 168833,3 tonne de thonidés a été débarquée au port d'Abidjan l'année 2006 et $S$. tritor représente les $70 \%$ (Diaha et al., 2009). Elle est aussi très appréciée pour son goût par les populations locales. Cependant, en Afrique de l'Ouest, très peu d'informations sont d'une manière générale disponibles sur sa biologie et particulièrement sur ses habitudes alimentaires. En effet, celles-ci demeurent assez rares et fragmentaires. En Côte d'Ivoire, les seules informations existantes, sont celles d'ordre général pour les thonidés mineurs. Ce sont des espèces carnivores qui se nourrissent principalement de Clupeidae (Johnson et Gill, 1998). Quant aux travaux effectués dans les autres régions de l'Afrique de l'Ouest, ils se résument le plus souvent en un simple inventaire des proies ingérées (Bard et al., 1988; Bahou et al., 2007).

L'objectif de cette étude est de caractériser le régime alimentaire de Scomberomorus tritor, de décrire l'étendu de sa variabilité temporelle et de mettre en évidence les relations qui peuvent exister avec la taille des individus capturés dans le golfe de Guinée et débarqués au port d'Abidjan.

\section{MATERIEL ET METHODES \\ Zone d'étude}

Le golfe de Guinée (Figure 1) est situé en Afrique de l'Ouest, au sein du grand écosystème marin que représentent les régions côtières. Il est délimité au Nord par la côte Ouest africaine, au Sud par l'équateur, à

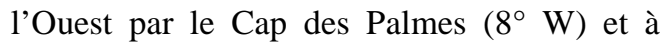
l'Est par $2^{\circ} 30 \mathrm{E}$ (Pezennec et Bard, 1992). Le climat est du type guinéen et on y rencontre une série de plages sablonneuses qui forment une large voûte ouverte à l'océan Atlantique.

Sa particularité majeure est la présence de quatre saisons marines déterminées par la variation de la température au cours de l'année (Pezennec et Bard, 1992). Ainsi les deux saisons froides (une grande de juillet à octobre et une petite de janvier à février) sont caractérisées par une résurgence d'eau océanique froide et salée (salinité supérieure à $35^{\circ} \%$ ) et une température faible comprise entre 23 et $25{ }^{\circ} \mathrm{C}$. Les saisons chaudes sont également au nombre de deux (une grande de mars à juin et une petite de novembre à décembre), et elles sont définies par les eaux océaniques provenant du large avec une salinité inférieure à $35^{\circ} \%$ et une température élevée comprise entre 28 et $30^{\circ} \mathrm{C}$ (Golé Bi et al., 2005).

\section{Stratégie d'échantillonnage et d'analyse des contenus stomacaux}

L'échantillonnage ichtyologique a été réalisé mensuellement (de janvier 2006 à janvier 2008) au port d'Abidjan, lors des débarquements des chalutiers. Le chalut pélagique a été l'engin utilisé par cette unité de pêche. La marée pour ces chalutiers a été estimée à 5 jours. Durant les premières semaines de chaque mois, une caisse de $S$. tritor a été échantillonnée de manière aléatoire selon l'état de fraicheur des spécimens. Chaque individu a été ensuite pesé à $0,1 \mathrm{~kg}$ près et la longueur à la fourche (LF) mesurée au centimètre près (extrémité de la mâchoire inférieure jusqu'au creux de la fourche de la nageoire caudale). La partie comprise entre l'œsophage et la première boucle intestinale (bulbe stomacal) a été détachée et conservée dans une solution de formol à $5 \%$ pour être examinée ultérieurement.

$\mathrm{Au}$ laboratoire les estomacs ont été disséqués et le contenu rincé à l'eau sur une série de tamis de mailles $1000 \mu \mathrm{m} ; 500 \mu \mathrm{m}$; $250 \mu \mathrm{m}$ et $100 \mu \mathrm{m}$, avant d'être examiné à l'œil nu ou à la loupe binoculaire suivant la taille des individus proies. Les différentes proies ont été triées, dénombrées, pesées et identifiées. A partir des données recueillies, ont été calculés le coefficient de vacuité $(\mathrm{CV})$, trois pourcentages $\mathrm{Cn} \%, \mathrm{Cp} \%$ et $\% \mathrm{~F}$ et deux indices alimentaires $\mathrm{Q}$ et IRI proposés par Rosecchi et Nouaze (1987).

- Coefficient de vacuité (CV)

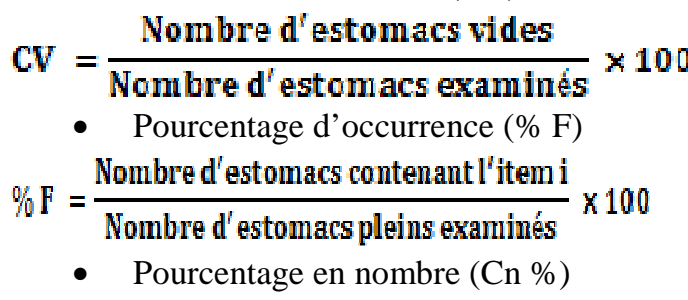




$$
\begin{aligned}
& \mathrm{Cn} \%=\frac{\text { Nombre d'individus d'un item } \mathrm{i}}{\text { Nombre total de proie }} \times 100 \\
& \text { - Le pourcentage en poids (Cp \%) } \\
& \mathrm{Cp} \%=\frac{\text { Poids d' un item } 1}{\text { Poids total des proies }} \times 100
\end{aligned}
$$
l'item (IRI) (Pinkas et al., 1971) et le coefficient alimentaire (Q) (Hureau, 1970) ont été utilisés pour avoir une approche plus sensible du régime alimentaire de Scomberomorus tritor.

- Indice d'importance relative (IRI) (Pinkas et al., 1971)

IRI $=\% \mathbf{F}(\mathbf{C n} \%+\mathbf{C p} \%)$

L'expression en pourcentage est de la forme:

$$
\% \text { IRI }=\frac{\text { IRI }}{\sum \text { IRI }} \times 100
$$

La classification des proies en thèmes d'aliments préférentiels, secondaires et accidentelles a été faite selon la méthode proposée par Rosecchi et Nouaze (1987). La valeur de l'indice de chaque item a été exprimée en pourcentage de la somme de tous les indices puis classée par ordre de rang décroissant. En partant de la proie de rang 1 on a additionné les indices de chacune des proies de la première à la nième jusqu'à obtenir 50\%, ou plus, de l'indice total. Ces proies sont appelées préférentielles. Ce calcul se poursuit jusqu'à obtenir $75 \%$ ou plus. Ces dernières sont alors qualifiées de proies secondaires. Enfin les dernières proies de la liste sont considérées comme accessoires ou accidentelles.

- Coefficient alimentaire Q (Hureau, 1970) :

\section{$\mathrm{Q}=\mathrm{Cn} \% \times \mathbf{C p} \%$}

La classification a été effectuée selon la méthode de Hureau (1970). Si (Q > 200), les proies sont qualifiées de préférentielles; si (20<Q $<200)$, les proies sont secondaires ; et si $(\mathrm{Q}<20)$, les proies sont accessoires.

Des classes de taille des spécimens échantillonnés ont été déterminées sur la base de la règle de Sturge (Scherrer, 1984):

$$
\begin{aligned}
& \text { - Nombre de classe (NC): } \\
& \mathrm{NC}=1+\mathbf{3}, \mathbf{3} \log _{10}(\mathrm{~N})
\end{aligned}
$$

Où $\mathrm{N}$ : est le nombre total des spécimens et NC: nombre de classe.

$$
\begin{aligned}
& \text { - Intervalle de classes (I) } \\
& \mathbf{I}=\text { (LF } \max -\text { LF min) } / \text { NC }
\end{aligned}
$$

Où LF min: la plus petite longueur à la fourche ; LF max: la plus grande longueur à la fourche.

Ces données obtenues ont été soumises à une analyse de classification hiérarchique ascendante, afin de pouvoir regrouper les classes de tailles dont les coefficients de vacuité ou les régimes sont plus proches. Certaines classes ont été également regroupées compte tenu de leur faible effectif enregistré.

\section{Analyses statistiques \\ Une analyse des classifications} hiérarchiques ascendantes a été réalisée par la méthode de Ward du logiciel Statistica 1.7 à partir de la matrice «Indice d'importance relative- échantillons des classes de tailles». Le test statistique du coefficient de rang de Spearman (rs) du logiciel $\mathrm{R}$ (The $\mathrm{R}$ Foundation for Statistical Computing Version 2.0.1) réalisé sur les pourcentages de vacuité des estomacs a été utilisé pour comparer les modes de vacuité en fonction des saisons marines et en fonction des classes de tailles. Ce coefficient a également permis de comparer les régimes selon les classes de tailles et les saisons marines afin d'illustrer les différences et les ressemblances de régime alimentaire entre les populations de S. tritor. $\mathrm{Si}$ rs $=1$, les régimes sont rigoureusement identiques, si rs $=0$, les régimes sont indépendants et si rs $=-1$, les régimes sont rigoureusement inverses (Scherrer, 1984). Le test de Kruskal-Wallis du logiciel $\mathrm{R}$ a été effectué sur les coefficients de vacuité en fonction $\mathrm{du}$ sexe pour déterminer les différences significatives $(\mathrm{p}<0,05)$.

\section{RESULTATS}

Variation du coefficient de vacuité en fonction du sexe

Un effectif total de 832 estomacs a été examiné les années 2006 - 2007 et 220 étaient vides. Le coefficient de vacuité $(\mathrm{Cv})$ 
calculé à cet effet a été de 26,44\%. Les coefficients de vacuités des deux populations (mâles (M) et femelles (F)), n'étant pas statistiquement différents (Kruskal-Wallis entre $(\mathrm{M})$ et $(\mathrm{F}), \mathrm{p}=0,24>0,05)$, ils ont été regroupés pour constituer l'échantillon $(\mathrm{M}+\mathrm{F})$. Sa représentation graphique (Figure 2) montre les pourcentages les plus élevés les mois de mai et juin avec $15,03 \%$ et $17,34 \%$.

\section{Variation du coefficient de vacuité en} fonction des saisons marines

La Figure 3 donne l'évolution du coefficient de vacuité de $S$. tritor en fonction des saisons marines dans le golfe de Guinée. Les spécimens pêchés pendant les saisons froides ont enregistré les plus faibles pourcentages du coefficient de vacuité (petite saison $(13,97 \%)$ et grande saison $(10,3 \%))$. La différence entre les coefficients de ces deux saisons froides est significative $(\mathrm{p}=0,035<$ $0,05)$. Pendant la grande saison chaude, le pourcentage de vacuité est très élevé $(46,76 \%)$ et significativement différent $(p=0,004<$ $0,05)$ de celui de la grande saison froide $(10,3 \%)$. Cette différence est également significative $(\mathrm{p}=0,034<0,05)$ entre la petite saison chaude $(28,96 \%)$ et la petite saison froide $(13,97 \%)$.

\section{Variation du coefficient de vacuité en fonction de la taille}

La longueur à la fourche des spécimens examinés est comprise entre 14114. Dix classes de tailles ont été déterminées selon la règle de Sturge. En raison du faible effectif des classes 1 et 2 ( 1 et 14 spécimens) et $7,8,9,10(5 ; 1 ; 2$ et 1 spécimen respectivement), les classes 1 et 2 ont été fusionnées, de même que les classes 7, 8, 9 et 10 (Tableau 1). La représentation graphique du coefficient de vacuité en fonction de ces classes de tailles (Figure 4) montre une nette fluctuation pour la classe $3(44$ à $54 \mathrm{~cm})$. Le coefficient de vacuité des autres classes étant relativement faible. Pour vérifier l'existence d'une similitude éventuelle, une classification hiérarchique a été effectuée. Le dendrogramme obtenu à partir de ces classes de tailles indique deux groupes (Figure 5). Le premier comprend tous les individus de tailles inférieures à $44 \mathrm{~cm}$ et ceux de tailles supérieures à $54 \mathrm{~cm}(\mathrm{n}=102)$. Le second regroupe uniquement ceux de taille appartenant à l'intervalle (44 à $54 \mathrm{~cm}),(\mathrm{n}=$ 118). Le test de corrélation de rang de Spearman effectué entre les deux groupes est significatif $(\mathrm{p}<0,05 ; \mathrm{rs}=0,67)$.

\section{Profil général du régime alimentaire}

Sur ce total de 832 estomacs de $S$. tritor provenant du golfe de Guinée examinés, 612 contenaient des aliments. Dix catégories d'aliments repartis entre quatre groupes ont été identifiées (Tableau 2). Il s'agit des Poissons, Céphalopodes, Crustacés et les débris regroupés sous le terme de «autres aliments ». Le groupe des poissons est constitué de trois familles (Clupeidae, Engraulididae et Carangidae) au sein desquelles se trouvent cinq espèces $(2,1$ et 2 respectivement). Les Céphalopodes comprennent deux familles (Octopodidae et Sepiidae) qui ne comportent qu'une seule espèce chacune. Les espèces de crustacés sont au nombre de deux, regroupées également au sein de deux familles.

D'après la classification selon le coefficient alimentaire (Tableau 2), les poissons sont les proies préférentielles $(\mathrm{Q}=$ 7596,73; Q > 200), suivi des proies accessoires constituées de céphalopodes et de crustacés dont les coefficients sont respectivement $(\mathrm{Q}=0,92$ et $\mathrm{Q}=0,63)$. Dans le groupe des poissons, la famille des Clupeidae a le coefficient le plus élevé $(\mathrm{Q}=$ 2580,83), ensuite les Engraulididae $(\mathrm{Q}=$ $479,64)$ et enfin les Carangidae $(Q=19,65)$. Les espèces fortement sollicitées au sein de chaque familles, sont Sardinella maderensis (Lowe, 1839), Engraulis encrasicolus (Linnaeus, 1758) et Caranx senegalus (Cuvier, 1833). Au niveau des céphalopodes, et des crustacés ce sont respectivement les espèces Octopus defilippi (Verny, 1851) et Skyonia galeata (Holthuis, 1952) qui sont prépondérantes. Le groupe «Autres aliments» $(\mathrm{Q}=41,94)$ devrait en principe se situer dans 
les proies accessoires mais, compte tenu de la diversité des éléments regroupés en son sein, il n'a pas été classé.

L'analyse de l'indice d'importance relative indique également les poissons comme proies préférentielles (IRI $=94,25 \%$ ). De toutes les espèces de poissons, $S$. maderensis est la plus consommée (IRI = $75,46 \%$ ) suivi de E. encrasicolus (IRI = $17,17 \%$ ), ensuite de $S$. aurita (IRI $=0,82 \%$ ), C. senegalus $($ IRI $=0,75 \%)$ et enfin $S$. dorsalis (IRI $=0,05 \%)$. Les autres groupes taxonomiques (céphalopodes et crustacés) sont considérés comme des proies accidentelles (IRI $=0,4 \%)$.

Variation du régime alimentaire en fonction des saisons marines

Le groupe faunistique représenté dans le contenu stomacal de $S$. tritor a été analysé en fonction des 4 saisons marines (petite saison froide, grande saison chaude, grande saison froide et petite saison chaude) (Figure 6). En petite saison froide, les Clupeidae représentent les proies préférentielles $(55 \%)$ et les Engraulidae les proies secondaires (24\%). Les Carangidae (5\%), les Octopodidae (7\%), les Sepiidae (3\%), Sicyonidae $(5 \%)$ et les Penaeidae $(1 \%)$ constituent les proies accessoires avec des pourcentages inférieurs à $10 \%$.

Pendant la grande saison chaude, ils se nourrissent principalement de (58\%) Clupeidae et en second lieu d'Engraulididae (31\%). Les autres familles sont des aliments accessoires avec des IRI inférieurs à $5 \%$. Durant la grande saison froide, la consommation est focalisée sur les Clupeidae qui constituent alors les proies principales (56\%). Les Engraulididae (30\%) sont les proies secondaires et les autres familles les proies accessoires. Pendant la petite saison chaude, ce sont les Engraulididae qui constituent les proies principales (67\%) et les Clupeidae (18\%) les proies secondaires. Les autres familles représentées par les Carangidae, les céphalopodes et les crustacés demeurent les proies accessoires.
La corrélation entre les pourcentages indiciaires des items, obtenus aux trois saisons marines n'est pas significative $(\mathrm{p}=0,95 ; \mathrm{p}$ > 0,05). Il y a alors une similitude des régimes pendant la petite saison froide, la grande saison chaude et la grande saison froide. Ce qui n'est pas le cas pour la petite saison chaude où la différence est significative.

\section{Variation du régime alimentaire en fonction de la taille}

Le nombre de classe déterminé sur la base de la règle de Sturge, est de 8 avec un intervalle de $10 \mathrm{~cm}$ (Tableau 3). Le dendrogramme obtenu (Figure 7), a permis de regrouper ces classes en deux grands groupes. Le groupe 1 renferme tous les individus de longueur à la fourche inférieure à $62 \mathrm{~cm}$ et constitué des 4 premières classes de tailles. Le groupe 2 renferme les individus de longueur à la fourche supérieure ou égale à $62 \mathrm{~cm}$ et comprenant les classes de tailles 5, 6 et 7. La Figure 8 qui représente l'indice d'importance relative de $S$. tritor en fonction de la taille, montre que les spécimens du groupe 1 ont pour aliments principaux les Clupeidae $(80,44 \%)$ avec pour espèces principales $S$. maderensis et $S$. aurita. Cette dernière espèce présente en revanche un IRI égal à $0,92 \%$ contre $75,72 \%$ pour les S. maderensis. Les proies secondaires sont les Engraulididae $(18,37 \%)$ et les Carangidae $(0,83 \%)$. Les autres constitués des débris d'animaux, des céphalopodes et de crustacés sont les proies accidentelles. Ceux du groupe 2, qui ont pour aliments préférentiels les Clupeidae et les Engraulididae avec pour IRI respectif $45,14 \%$ et $47,55 \%$. L'aliment secondaire est représenté par les autres familles que sont les débris d'animaux, Carangidae, les céphalopodes et les Crustacés qui ont des pourcentages d'IRI inférieurs à un. Le coefficient de corrélation de rang de Spearman calculé à partir des pourcentages d'IRI des deux groupes est significatif ( $\mathrm{rs}=$ $0,78 ; \mathrm{p}=0,001)$. 


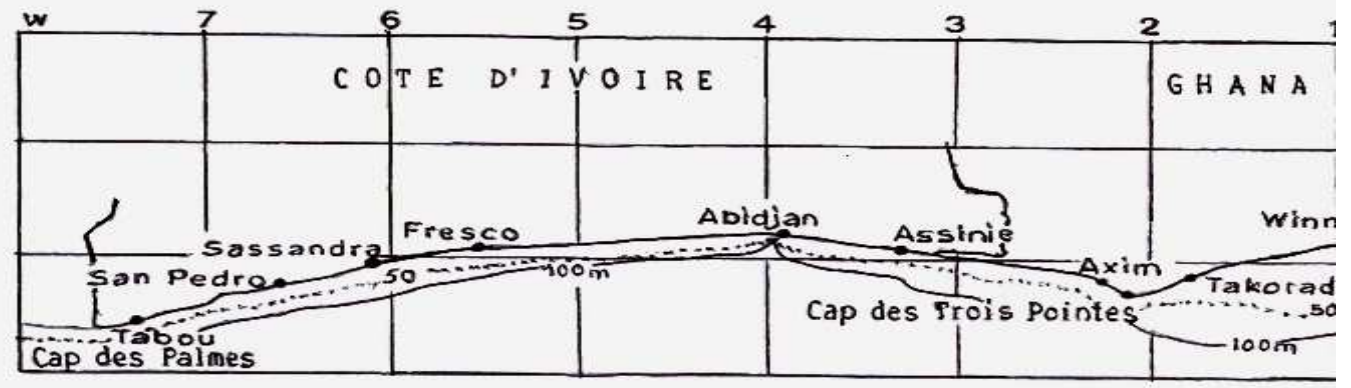

0

$100 \mathrm{~km}$

Figure 1: Situation géographique de la zone de pêche dans le golfe de Guinée

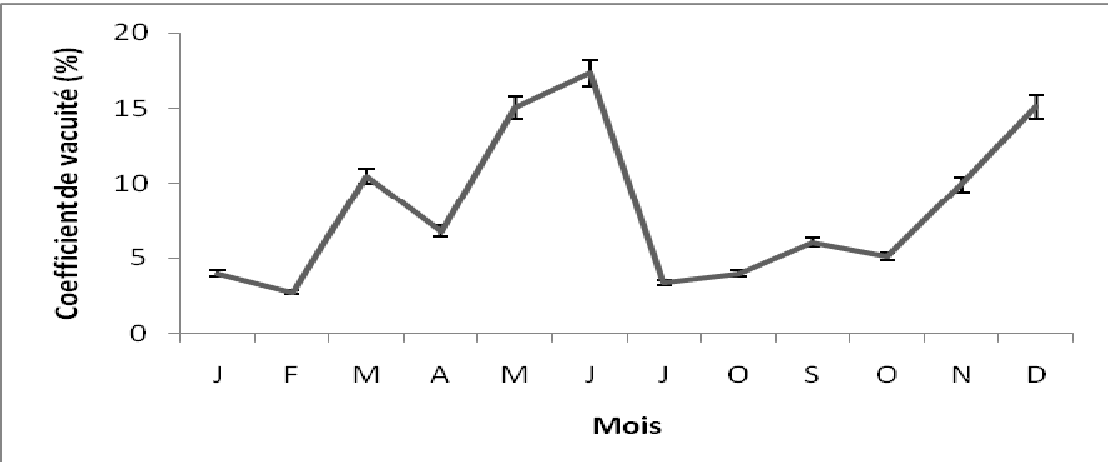

Figure 2: Evolution mensuelle du coefficient de vacuité $(\mathrm{Cv})$ chez Scomberomorus tritor.

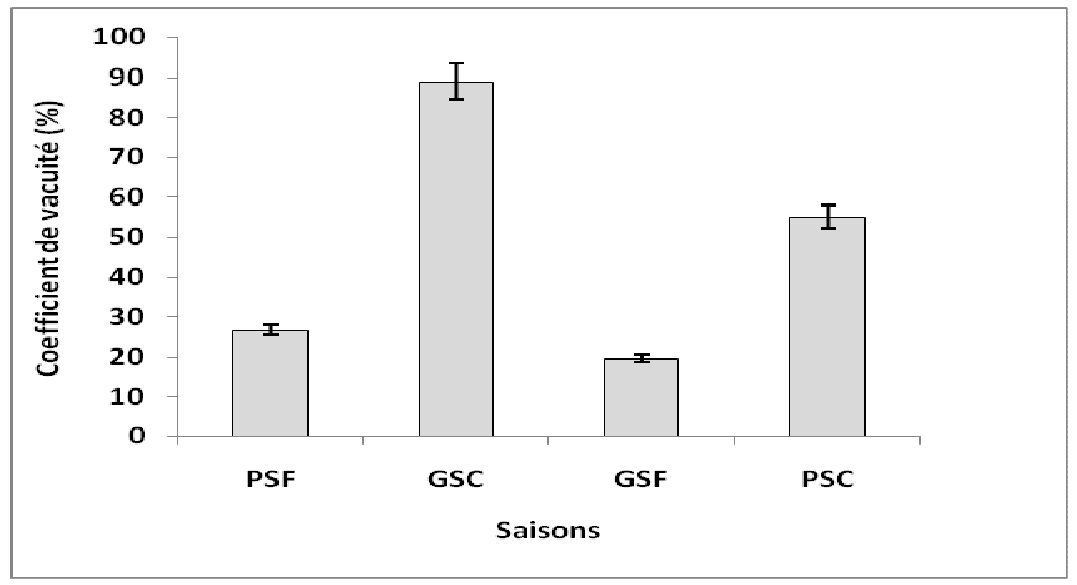

Figure 3: Evolution du coefficient de vacuité en fonction des saisons.

PSF (petite saison froide); GSC (grande saison chaude); GSF (grande saison froide); PSC (petite saison chaude). 


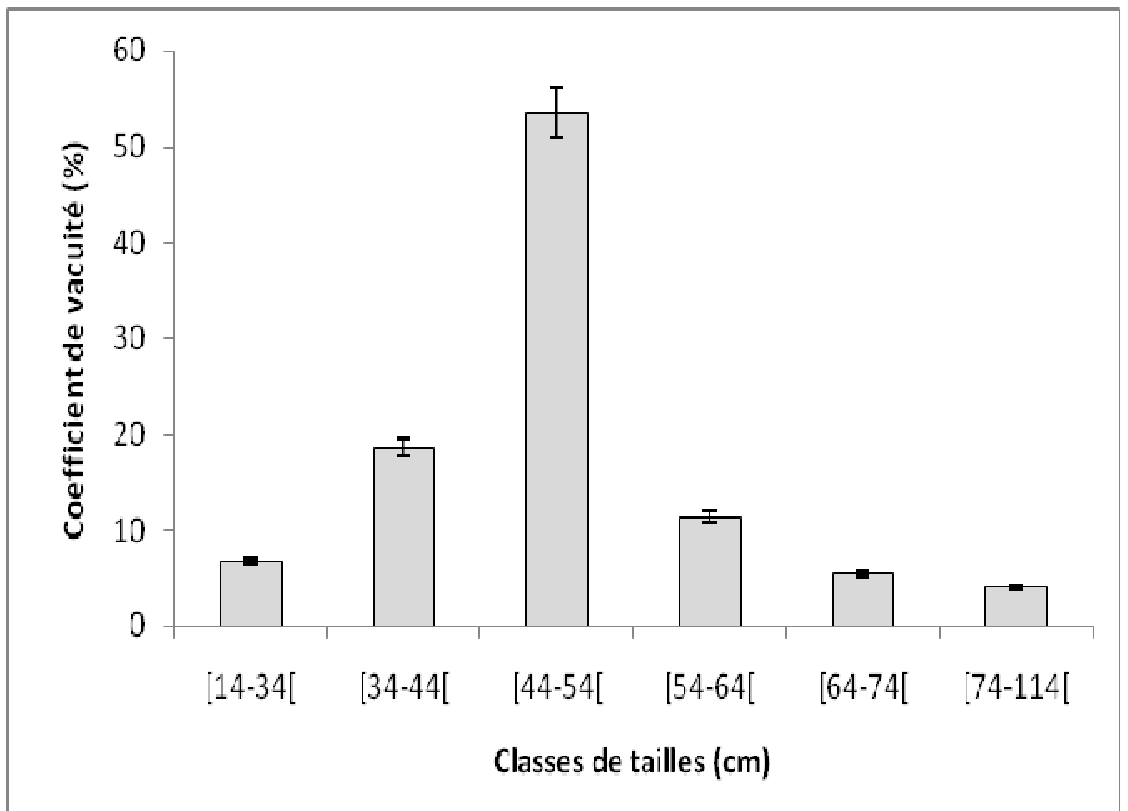

Figure 4: Evolution du coefficient de vacuité en fonction de six classes de tailles de Scomberomorus tritor dans le golfe de Guinée.

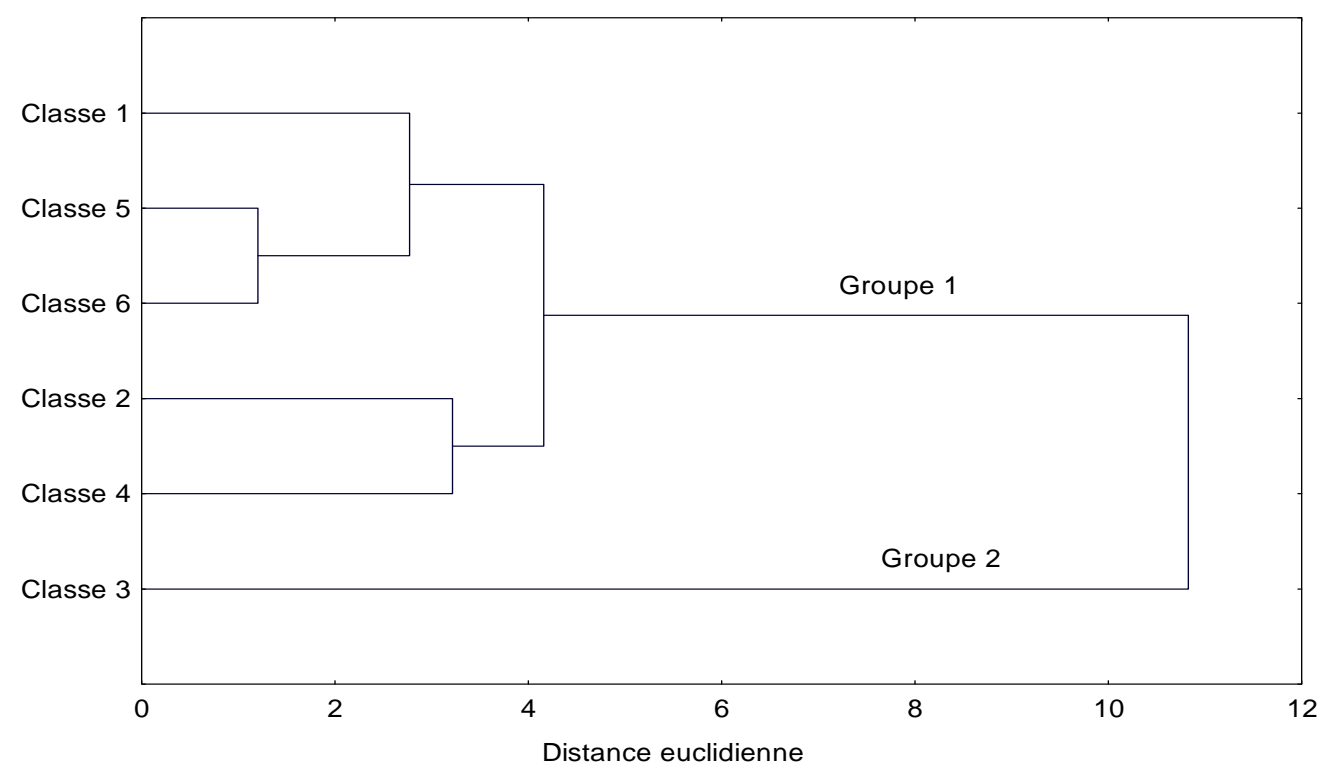

Figure 5: Dendrogramme montrant les similarités de vacuité entre les échantillons de six classes de tailles de Scomberomorus tritor provenant du golfe de Guinée (Côte d'ivoire).

Analyse effectuée sur la base de la distance euclidienne et la méthode de Ward. 


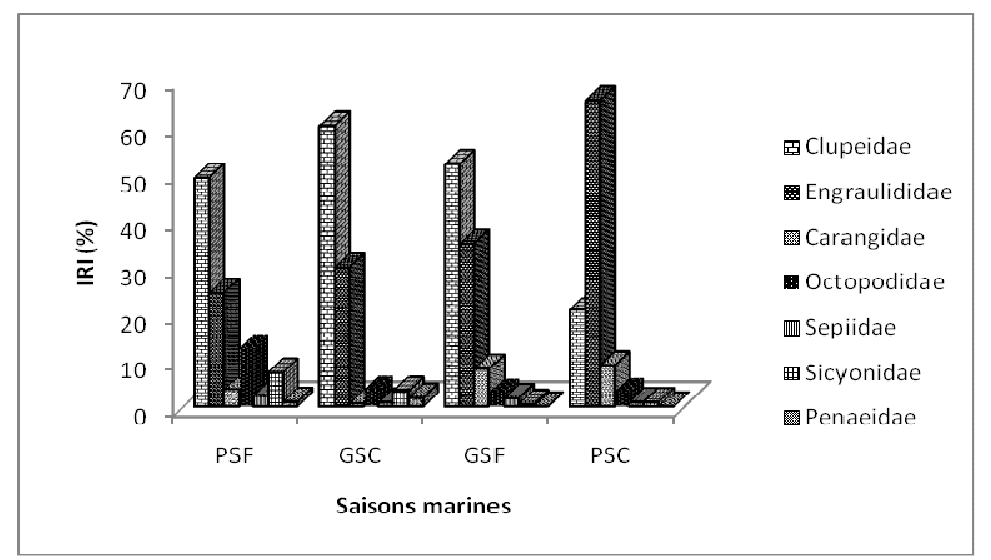

Figure 6: Variation Régime alimentaire en fonction des saisons marines: pourcentage des indices d'importance relative (IRI) par famille et par saison des différentes proies ingérées par Scomberomorus tritor.

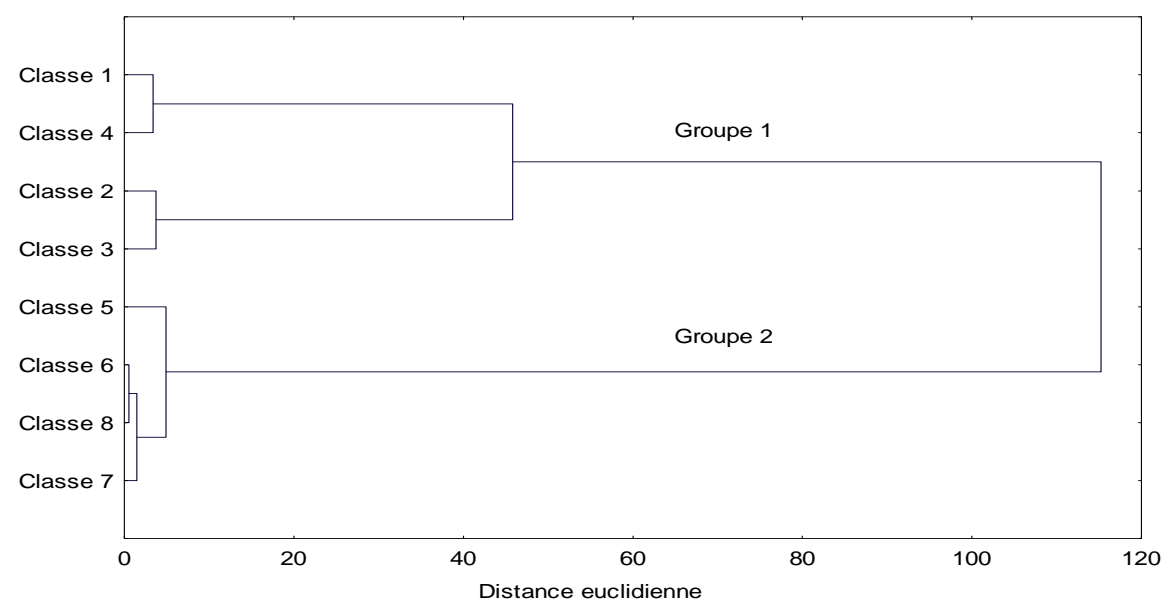

Figure 7: Dendrogramme montrant les similarités alimentaires entre les échantillons de huit classes de tailles de Scomberomorus tritor provenant du golfe de Guinée (Côte d'Ivoire).

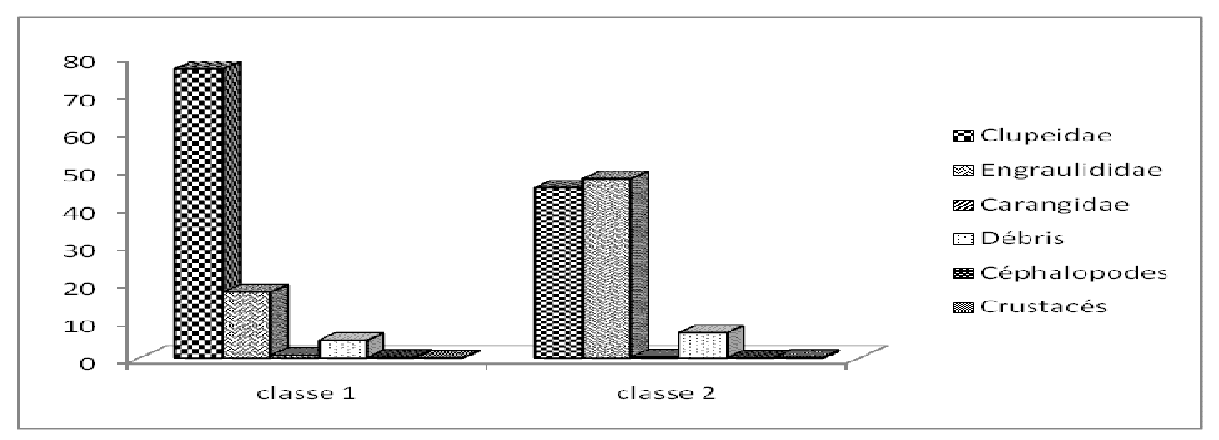

Figure 8: Variation du régime alimentaire (IRI) de Scomberomorus tritor en fonction de la taille. Classe 1: $\mathrm{LF}<53 \mathrm{~cm}$; Classe 2: $\mathrm{LF} \geq 53 \mathrm{~cm}$. 
Tableau 1 : Différentes classes de taille de Scomberormorus tritor obtenues par la règle de Sturge et effectifs correspondants dans le golfe de Guinée.

\begin{tabular}{llll}
\hline Classes & Etendue & Effectif & Groupe \\
\hline 1 & {$[14-34[$} & 15 & \\
2 & {$[34-44[$} & 41 & 1 \\
4 & {$[54-64[$} & 25 & \\
5 & {$[64-74[$} & 12 & \\
6 & {$[74-114[$} & 9 & \\
\hline 3 & {$[44-54[$} & 118 & 2 \\
\hline \multicolumn{4}{l}{ Regroupements obtenus à partir de la classification ascendante de la Figure 5. }
\end{tabular}

Tableau 2: Composition du régime alimentaire de Scomberomorus tritor dans le golfe de Guinée et pourcentages des indices alimentaires correspondants.

\begin{tabular}{|c|c|c|c|c|c|}
\hline Aliments & Cn & $\mathbf{C p}$ & $\mathbf{f}$ & IRI \% & Q \\
\hline $\begin{array}{l}\text { Poissons } \\
\text { Clupeidae }\end{array}$ & & & & & \\
\hline Sardinella maderensis (Lowe, 1839) & 37,16 & 68,74 & 41,52 & 75,46 & 2554,38 \\
\hline Sardinella aurita (valencienne, 1847) & 3,99 & 6,63 & 4,5 & 0,82 & 26,45 \\
\hline $\begin{array}{l}\text { Engraulididae } \\
\text { Engraulis encrasicolus (Linnaeus, 1758) }\end{array}$ & 32,92 & 14,57 & 21,06 & 17,17 & 479,64 \\
\hline $\begin{array}{l}\text { Carangidae } \\
\text { Caranx senegalus (Cuvier, 1833) } \\
\text { Selene dorsalis (Gill, 186e) }\end{array}$ & $\begin{array}{l}3,87 \\
0,75\end{array}$ & $\begin{array}{l}4,71 \\
1,89\end{array}$ & $\begin{array}{l}5,11 \\
1,02\end{array}$ & $\begin{array}{l}0,75 \\
0,05\end{array}$ & $\begin{array}{l}18,23 \\
1,42\end{array}$ \\
\hline $\begin{array}{l}\text { Céphalopodes } \\
\text { Octopodidae } \\
\text { Octopus defilippi (Verny, 1851) }\end{array}$ & 3,74 & 0,17 & 3,47 & 0,23 & 0,64 \\
\hline $\begin{array}{l}\text { Sepiidae } \\
\quad \text { Sepia elegans (Blainville, 1827) }\end{array}$ & 1,12 & 0,02 & 1,44 & 0,03 & 0,02 \\
\hline $\begin{array}{l}\text { Crustacés } \\
\quad \text { Sicyonidae } \\
\quad \text { Skyonia galeata (Holthuis, 1952) }\end{array}$ & 2 & 0,16 & 2,45 & 0,09 & 0,32 \\
\hline $\begin{array}{l}\text { Penaeidae } \\
\text { Parapenaeopis atlantica (Balss, 1914) }\end{array}$ & 0,62 & 0,08 & 0,81 & 0,01 & 0,05 \\
\hline $\begin{array}{l}\text { Autres aliments } \\
\text { Débris d'animaux } \\
\text { Total }\end{array}$ & 13,84 & 3,03 & 18,61 & 5,39 & 41,94 \\
\hline $\begin{array}{l}\text { Poissons } \\
\text { Céphalopodes } \\
\text { Crustacés } \\
\text { Autres aliments }\end{array}$ & $\begin{array}{l}78,69 \\
4,86 \\
2,62 \\
13,84\end{array}$ & $\begin{array}{l}96,54 \\
0,19 \\
0,24 \\
3,03\end{array}$ & $\begin{array}{l}73,21 \\
4,49 \\
3,89 \\
18,61\end{array}$ & $\begin{array}{l}94,25 \\
0,28 \\
0,12 \\
5,39\end{array}$ & $\begin{array}{l}7596,73 \\
0,92 \\
0,63 \\
41,94\end{array}$ \\
\hline
\end{tabular}

Cn \%: pourcentage numérique; Cp \%: pourcentage pondéral; \% F: pourcentage d'occurrence; IRI: pourcentage d'indice d'importance relative; Q: coefficient alimentaire 
Tableau 3: Différentes classes de tailles obtenues par la règle de Sturge et leurs effectifs correspondants. Regroupements obtenus à partir de la classification hiérarchique ascendante du pourcentage de l'indice d'importance relative (IRI) de S. tritor du golfe de Guinée (Côte d'ivoire).

\begin{tabular}{llll}
\hline Classes & Etendue & Effectif & Groupe \\
\hline 1 & {$[14-34[$} & 60 & \\
2 & {$[34-44[$} & 180 & 1 \\
3 & {$[44-54[$} & 254 & \\
4 & {$[54-64[$} & 69 & \\
\hline 5 & {$[64-74[$} & 23 & \\
6 & {$[74-84[$} & 7 & \\
7 & {$[84-94[$} & 11 & 2 \\
8 & {$[94-114[$} & 8 & \\
\hline
\end{tabular}

\section{DISCUSSION}

L'état frais des proies contenues dans la plus part des estomacs examinés traduirait une alimentation journalière de cette espèce. Cette assertion confirme les observations des auteurs ayant travaillés sur les thonidés en générale et sur la thonine en particulier (Bahou et al., 2007; Hattour, 2008). Le coefficient de vacuité est élevé. Cela peut être dû au stress créé au niveau du poisson lors de leur capture avec les chaluts pélagiques. Une régurgitation des proies contenues dans leur estomac peut avoir eu lieu, comme c'est le cas chez d'autres espèces notamment chez un carangidae (Trachinotus teraia) dans la lagune Ebrié en Côte d'Ivoire (Sylla et al., 2008). Cette vacuité importante peut aussi laisser supposer que pendant les périodes de pourcentages élevés, les poissons ne se nourrissaient pas convenablement. L'activité alimentaire serait alors influencée par les conditions du milieu et par l'état physiologique des poissons. L'étude de la reproduction de $S$. tritor a montré que la période de ponte qui se déroule entre mai et juin, est également la période où le coefficient de vacuité atteint son maximum. En effet, l'augmentation du poids des gonades peut comprimer le tube digestif, réduire le bol alimentaire et amener les poissons à se nourrir moins (Layachi et al., 2007). La vacuité faible pendant les saisons froides pourrait aussi être en relation avec la disponibilité alimentaire. En effet, avant et après la ponte qui à lieu dans les mois de mai et juin, $S$. tritor se nourrirait davantage afin de mener à terme le développement des gonades d'une part, et d'autre part pour récupérer de l'énergie et se préparer pour le cycle de reproduction suivant (Layachi et al., 2007).

Par ailleurs, la classification des items selon les deux méthodes (l'indice d'importance relative: IRI et le coefficient alimentaire: Q) permet d'affirmer que $S$. tritor se nourrit principalement de poissons dont les espèces principales sont Sardinella maderensis et Engraulis encrasicolus appartenant respectivement à la famille des Clupeidae et des Engraulididae. Ces résultats concordent avec ceux trouvés dans l'estuaire du Sine-Saloum au Sénégal (Ngouda, 1997) et ceux trouvés dans l'Atlantique (Froese et al., 2003; Johnson et Gill, 1998). C'est une espèce qui ne s'attaque qu'aux proies dont la taille est plus ou moins adaptée au calibre de leur bouche (Yatabary, 1983). Des résultats similaires ont été trouvés par d'autres auteurs dans l'atlantique (Iccat, 2009). Les proies accidentelles rencontrées sont également constituées des céphalopodes et les crustacés. Le spectre alimentaire de cette espèce est donc réduit par rapport aux autres espèces de thonidés mineurs. En effet, des travaux réalisés sur les autres espèces de thonidés 
mineurs telles qu'Euthynnus alletteratus et Scomber japonicus (Hattour, 2008), ont montré une gamme de proies plus large avec plusieurs espèces de poissons. Ce spectre alimentaire de $S$. tritor est par contre identique à celui de l'espèce Sarda sarda dans les eaux tunisiennes (Hattour, 2008).

L'analyse du régime alimentaire de $S$. tritor en fonction des saisons montre que les Clupeidae et les Engraulididae sont les proies principales en toutes saisons. Cependant, durant la petite saison chaude, la proportion d'Engraulididae augmente significativement par rapport à celle des Clupeidae qui diminue. Cette différence dans la disponibilité serait imputable à l'upwelling qui engendre les conditions nécessaires à la prolifération de certaines proies par rapport à d'autres.

Nos résultats montrent aussi que l'importance relative des deux principaux groupes de proies varie en fonction de la taille des poissons. En effet les spécimens de petites tailles (groupes 1) préfèrent les Clupeidae, en revanche, ceux de grandes tailles (groupe 2) consomment les Clupeidae et les Engraulididae équitablement. Ces résultats ne concordent pas avec ceux Naughton et Saloman (1981) et Bowman et al. (2000), qui trouvent une préférence au niveau des Engraulidae chez les petites tailles que chez les adultes dans l'Atlantique. Cette différence peut être due à une distribution préférentielle de ces proies dans les différents milieux d'étude.

D’une façon générale, S. tritor serait prédisposé à ingérer des proies de plus en plus grandes au fur et à mesure que sa taille augmente. Cette différence ontogénique dans les habitudes alimentaires n'est cependant pas spécifique à cette espèce de thonidé mineur, elle est également observée chez de nombreux autres poissons (Layachi et al., 2007). Selon cet auteur, ces changements ontogéniques impliquent presque toujours des choix de proies de grandes tailles susceptibles de fournir le maximum d'énergie aux prédateurs pour l'accomplissement des fonctions de croissance et de reproduction. Pour la couverture de ces besoins énergétiques, la recherche et la collecte de petites proies par les individus adultes s'avèrent plus coûteuses que celles de grandes tailles. Les proies qualifiées de divers, pourraient provenir, soit des estomacs des différents poissons ingérés, soit par des ingestions accidentelles (Yatabary, 1983).

\section{Conclusion}

Scomberomorus tritor est un poisson qui ne se nourrit pas convenablement pendant les périodes de reproduction. Son alimentation est principalement constituée de poissons dont les espèces dominantes sont Sardinella maderensis et Engraulis encrasicolus. Ce spectre alimentaire est le même toutes les saisons à l'exception de la petite saison chaude où il diffère à cause de la disponibilité des individus proies. Cette différence s'observe également au niveau de la taille. En effet, au fur et à mesure que la taille augmente, les individus de ces espèces acquièrent l'aptitude à ingérer des proies de plus en plus grandes.

\section{REFERENCES BIBLIOGRAPHIQUES}

Bahou L, Koné T, N'douba V, Kouassi NJ, Essetchi PK, Goore BG. 2007. Food composition and feeding habits of little tunny (Euthynnus alletteratus) in continental shelf waters of Côte d'Ivoire (West Africa). doi:10.1093/icesjms/fsm065, 1044-1052.

Bard FX, Cayre P, Diouf T. 1988. Les migrations. In Ressources, Pêche et Biologie des Thonidés Tropicaux de l'Atlantique Centre Est, Fonteneau A, Marcille J (eds). FAO Doc. Tech. Pêches; 111-156.

Bowman RE, Stillwell CE, Michaels WL, Grosslein MD. 2000. Food of North west Atlantic fishes and two common species of squid. NOAA Tech. Memo.

Collette BB, Nauen CE. 1983. Scombrids of the world. An annotated and illustrated catalogue of tunas, mackerels, bonitos and related species known to date. FAO Fish Synop., 125(2). 
Collette BB, Carol R, Barbara AB. 2001. Systematic of the Tunas and Mackerels Scombridae). In Tuna: Physiology, Ecology and Evolution. Fish. Physio., 19: $1-33$.

Diaha NC, N'da K, Soro Y. 2009. Etude comparée de la pêche des thonidés mineurs par les chaluts doubles et les pirogues dans la zone économique exclusive. Tropicultura, 27(3): 152-158.

Froese RD, Pauly DW. 2003. "Fish Base" (On-line). FishBase World Wide Web Electronic Publication.

Gole Bi GG, Koffi KJD, Dadi SG. 2005. Contribution socio-économique de la pêche artisanale en Côte d'Ivoire. DFID, Programme pour des Moyens d'Existence Durables dans la Pêche (PMEDP) en Afrique de l'Ouest (GCP/INT/735/UK).

Hattour A. 2008. Les thons mineurs tunisiens: Etude biologiques et pêche. SCRS/2008/055. Salammbô.

Hureau JC. 1970. Biologie comparée de quelques poissons antarctiques (Notothenüdae). Bull. Inst. Océanogr. Monaco, 68(1391).

Iccat. 2009. Manuel de l'ICAAT. Commission In publications ICCAT en ligne.

Johnson G, Gill A. 1998. Perches and their allies. In Encyclopedia of Fishes (2nd edn), Eschmeyer WN, Paxton JR (eds). Academic Press; 190.

Layachi M, Melhoaoui M, Ramdani M, Srour A. 2007. Etude préliminaire du régime alimentaire du Rouget-barbet (Mullus barbatus L., 1758) de la côte nord-est méditerranéenne du Maroc (Nador) au cours de l'année 2001 (poissons, Mullidae). Bul. Inst. Scien., 29: 25- 41.

Naughton SP, Saloma CH. 1981. Stomach contents of juveniles of king mackerel (Scomberomorus cavalla) and Spanish mackerel (S. maculatus). Northeast Gulf Sci., 5(1): 71-74.

Ngouda S. 1997. Contribution à l'étude qualitative du régime alimentaire de quelques poissons dans l'estuaire du Sine-Saloum (Sénégal). C.R.O. Senegal.

Pezennec O, Bard FX. 1992. Importance écologique de la petite saison d'upwelling ivoiro-ghanéenne et changements dans la pêcherie de Sardinella aurita. Aquat. Living Resour., 5: 249-259.

Pinkas L, Oliphant MS, Iverson LK. 1971. Food habits of albacore, blue fin tuna and bonito in Californian waters. Calif. Fish. Game, 152: 1-105.

Rosecchi E, Nouaze Y. 1987. Comparaison de cinq indices alimentaires utilisés dans l'analyse des contenus stomacaux. Rev. Trav Inst. Sci. Pêch Mar., 49(3, 4) : 111123.

Scherrer B. 1984. Résultats des données. In Biostatique, Gaetan, Morin (eds). Louiseville : Canada; 850 p.

Sylla S, Atsé BC, Kouassi NJ. 2008. Régime alimentaire de Trachinotus teraia (Carangidae) dans la lagune Ebrié (Côte d'Ivoire). Cybium, 32(1): 81-87.

Yatabary NT. 1983. Contribution à l'étude du régime alimentaire de Synodontis schall (Bloch- Schneider, 1801) dans le delta central du fleuve Niger. Rev. Hydrobiol. Trop., 16(3): 277-286. 